\title{
Transformer winding modal parameter identification based on poly-reference least-square complex frequency domain method
}

\author{
Yaning $\mathrm{Li}^{1,}$, , Hong $\mathrm{Yu}^{2}$ and Xiaoyan $\mathrm{Zhu}^{1}$ \\ ${ }^{1}$ North China Electric Power University, Baoding, China \\ ${ }^{2}$ Yunnan Power Grid Electric Power Research Institute Co., Ltd.,kunming, China
}

\begin{abstract}
The modal parameter of transformer winding, as direct reflections of mechanical performance, is an important theoretical foundation in the field of transformer manufacturing and detection of winding condition based on vibration. To identify the modal parameters accurately, a modal experiment on a $10 \mathrm{kV}$ transformer winding was conducted in this paper. A PolyMAX method to identify winding natural frequency and damping ratio was proposed using the experiment to identify transformer winding modal parameters. And it uses modal confidence criterion to verify. The first four order modal parameters, including natural frequency, damping ratio were extracted from the vibration signal. The results demonstrate that all the natural frequencies are far from two times of exciting frequency, which indicates that the design is appropriate. The parameters verifies by MAC, which verifies the effectiveness of the proposed method in identifying modal parameters of transformer windings.
\end{abstract}

Keywords: transformer; winding; modal parameters; PolyMAX.

\section{Introduction}

Transformer is one of the most important equipment in power system, and the stability of power system is very important to the safety of power system. In normal operation of the transformer, the windings are excited by the electric power to generate vibration. If the natural frequency of the transformer winding is close to the excitation frequency of the axial electric power, the winding will produce a resonance phenomenon. Therefore, it is important to identify the natural frequency of the winding, which is of great significance to the design and manufacture of the transformer with the natural frequency of the winding. In addition, in the long run or short circuit impact, the winding may appear loose or deformation, and the winding mode parameters can be accurately identified and explored.

At present, the analysis of the inherent characteristics of the transformer windings is limited to a simple model of the winding. The model of spring - pad is used to calculate the [1-2], but the model parameters are difficult to be determined, and the simplified model and the actual transformer winding structure are quite different. The finite element modelling and simulation analysis of the transformer winding and the body mode of the transformer winding and the body of Li Yan, such as, has not been verified. Wang Hongfang et al. Study the effect of [4] on the nonlinear vibration characteristics of

a Corresponding author : hdliyaning@163.com

(C) 2016. The authors - Published by Atlantis Press 
windings, but the inherent characteristics of the structure are discussed. Li Hongkui et al. The excitation vibration of the transformer windings is carried out by [5]. But only the first order natural frequency of the winding is extracted, and the winding damping ratio and vibration mode are not given. Because of the complex structure of transformer winding, nonlinear strong, so the vibration signal contains noise interference. In the time domain or frequency domain, the identification method of modal parameter identification method is limited to the vibration signal alone, and the identification accuracy is limited, and is sensitive to noise.

In this paper, the excitation vibration of a $10 \mathrm{KV}$ transformer winding is excited, the PolyMAX method is used to identify the modal parameters of the transformer windings and the 4 order natural frequency and damping ratio are obtained. Finally, the MAC (modal confidence criterion) is used to verify the identification results.

\section{Basic principle}

\subsection{PolyMAX modal identification method}

The PolyMax modal identification method is used in this paper, also known as multi reference least square complex frequency domain method (Polyreference for complex frequency domain method), proposed by the Belgian B. Peeters and H. van der Auweraer professor in 2004, and is based on the least square estimation theory and the impulse response function and the poles and residues between the complex exponential relationship, and use the matrix of frequency response function as a fitting function in the frequency domain analysis method. The method is first by single point excitation multi-point vibration pickup system response measurement points and between the excitations is obtained the frequency response function, by the frequency response function and through the standard algorithm. Finally, we get required for the model parameters. At the same time, the method is not limited by the size of the damping, the intensity of the mode and the interference of the noise. Because the pulse function contains all the information of the mode, it can be used to identify all the modal parameters of the structure system. Basic calculation procedures are as follows:

$H(\omega)$ computing test system transfer function $H(\omega)$ obtained based on the measured input signals $x(t)$ and $y(t)$, as follows:

$$
[H(\omega)]_{l \times m}=\frac{[B(\omega)]_{l \times m}}{[A(\omega)]_{m \times m}}
$$

Wherein, $B(\omega)$ is a frequency domain representation of the output signal $y(t) ; A(\omega)$ to form a frequency domain output signal $x(t) ; m$ is the number of the reference channel input stimulus; ${ }^{l}$ is the total number of channels in response to an output.

If $B(\omega)$ and $A(\omega)$ can be expressed as follows:

$$
\begin{gathered}
{[B(\omega)]=\sum_{r=0}^{p} Z^{r}\left[\beta_{r}\right]_{l \times m}} \\
{[A(\omega)]=\sum_{r=0}^{p} Z^{r}\left[\alpha_{r}\right]_{m \times m}}
\end{gathered}
$$

For any frequency $\omega_{k}$, lists of equations as represented by (1) can be measured according to $[H(\omega)]$. If we take a different frequency, a sufficient number of lists (over determined) equation, it 
can polynomial coefficients $\left[\beta_{r}\right]$ and $\left[\alpha_{r}\right](r=0,1, \cdots, p)$ obtained by the least squares estimation of the numerator and denominator of the matrix to be determined.

$\left[\beta_{r}\right],\left[\alpha_{r}\right]$ are real-valued coefficients of each element, they are set to the complex is possible. $\left[\alpha_{r}\right]$ appears on the denominator of the equation is the nonlinear parameter identification problem. However, a certain degree of linearization, the variable of the linear least-squares estimation problem.

$\alpha_{r}(r=0,1, \cdots, N-1)$ in the denominator polynomial coefficients based on known and given the $\alpha_{N}=I$, and construct its adjoint matrix for eigenvalue decomposition, we can get the system poles and modal participation factors, as follows:

$$
\left[\begin{array}{ccccc}
0 & I & \cdots & 0 & 0 \\
0 & 0 & \cdots & 0 & 0 \\
\vdots & \vdots & \cdots & \vdots & \vdots \\
0 & 0 & \cdots & 0 & I \\
-\alpha_{0}^{T} & -\alpha_{1}^{T} & \cdots & -\alpha_{N-2}^{T} & -\alpha_{N-1}^{T}
\end{array}\right] V=V \Lambda
$$

$\Lambda$ characteristic value for the matrix (diagonal matrix), diagonal elements $\lambda_{i}(i=1,2, \cdots, p N)$ as follow:

$$
\begin{aligned}
& \lambda_{i}=e^{-p_{i} \Delta t} \text { or } e^{-p_{i}^{*} \Delta t} \\
& p_{i}, p_{i}^{*}=-\sigma_{i} \pm j \omega_{d i}
\end{aligned}
$$

Modal damping ratio as follows:

$$
\xi_{i}=\frac{\sigma_{i}}{\omega_{i}}=\frac{\sigma_{i}}{\sqrt{\sigma_{i}^{2}+\omega_{d i}^{2}}}
$$

In all matrices obtained polynomial coefficients $\mathrm{A}$ and $\mathrm{B}$, the frequency domain using the least squares method the modal shape, the fitting function as follows:

$$
H(\omega)=\sum_{r=1}^{N}\left(\frac{\psi_{r} l_{r}^{T}}{j \omega-p_{r}}+\frac{\psi_{r}^{*} l_{r}^{H}}{j \omega-p_{r}^{*}}\right)-\frac{L}{\omega^{2}}+U
$$

\subsection{Verification based on MAC}

After the test analysis is completed in order to prevent the introduction of false mode or the loss of real mode, it is necessary to verify the modal parameters in order to use MAC to verify the application. MAC is the correlation coefficient of two modal vectors $\psi_{1}$ and $\psi_{2}$, as follows:

$$
M A C=\frac{\left|\sum_{i=1}^{L} \psi_{i 1} \psi_{i 2}\right|}{\left(\sum_{i=1}^{L} \psi_{i 1} \psi_{i 1}\right)\left(\sum_{i=1}^{L} \psi_{i 2} \psi_{i 2}\right)}
$$

The modal vector is independent of each other, and the MAC value between the two real modes should be close to 0 . 


\section{Experiment and simulation}

\subsection{Experimental description}

In this paper, the high voltage winding of a power transformer with S9-630/10, the rated voltage of $10 \mathrm{kV}$, and the connecting group are Y0. In order to be able to simulate the mechanical characteristics of high voltage power transformer winding, the winding is the same as that of the power transformer. The transformer is carried out with a KISTLER8763 acceleration sensor, and the sensitivity is $2 \mathrm{mV} / \mathrm{g}$, which is arranged on the winding surface by the metal clamp. At the top of the winding, the B\&K4808 electromagnetic exciter is used to stimulate the winding. The excitation signal is white noise signal, and the bandwidth is $20 \mathrm{kHz}$, which is provided by the digital signal generator and connected to the electromagnetic exciter by the power amplifier. The electromagnetic exciter is used to measure the excitation signal and the experimental scene is shown in Fig. 1.

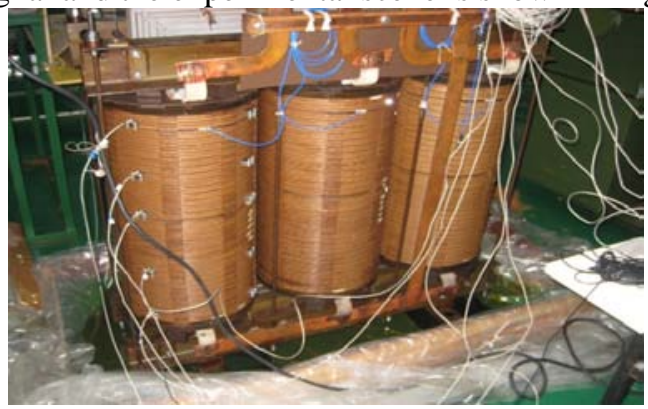

Figure 1. Overall view of transformer winding modal experiment

The experimental apparatus includes signal acquisition and analysis system, charge amplifier, vibration acceleration sensor, vibration sensor, power amplifier and signal generator.

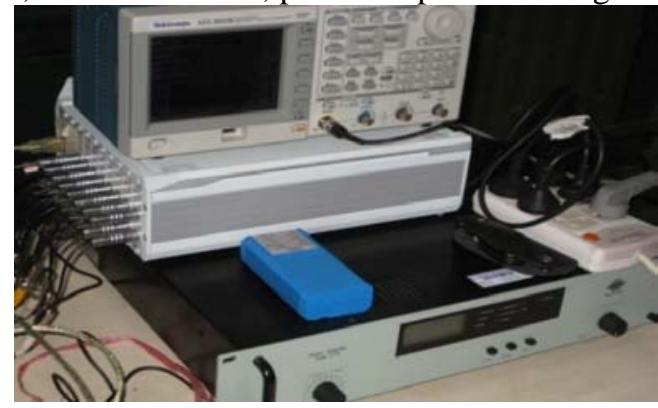

Figure 2. Signal generator, power amplifier and signal acquisition system

\subsection{Modal test of transformer winding}

In the interaction of the leakage magnetic field and current of the transformer windings, the electric power will be changed with the pulse of current and the magnetic field strength, which causes the winding vibration. When the sudden short circuit, the transformer winding is easy to produce the loose and the deformation under the huge short-circuit current. After the winding loose and the deformation will cause a greater imbalance electric excitation force, and form a vicious circle, the winding is damaged, even lead to serious accident of the transformer. Therefore, the capacity of the transformer is the key to the design of large capacity transformer.

Short circuit force can be classified as the axial force, radial to force and circumferntial force (of spiral winding), the role of axial force makes the winding in the axial direction is compressed, resulting in axial displacement and axial mechanical vibration; radial force makes the winding is compressed, winding stretch; circumferntial force the winding generates torsion effect. In the course 
of the short circuit, the transformer windings are generated under the action of short circuit force. The dynamic force in the winding is not only short of short circuit electromagnetic force, but also the friction force of inertia force, elastic force and displacement of the winding. The dynamic process is very complicated, which is influenced by the electromagnetic, mechanical, materials and other factors. In general, the calculation of the dynamic process of the transformer winding, the axial movement of the transformer winding under the axial force and the radial movement of the transformer under the action of the radial direction. Therefore, this study tests the axial mode and the radial direction of the transformer winding.

\subsubsection{Transformer winding axial modal test}

The purpose of the test is to measure the axial vibration of the winding, and the natural frequency and the corresponding vibration mode of the winding of the transformer are obtained. In the experiment, the white noise is used to motivate, and there are 20 vibration acceleration sensor measuring points. Fig. 3 is the location of the vibration sensor.

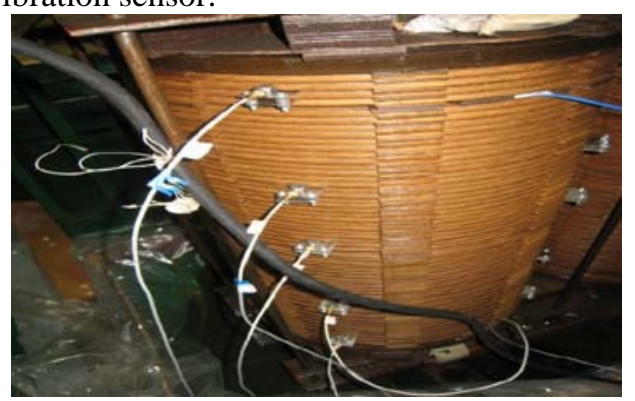

Figure 3. Vibration sensor location diagram

The exciting point is located in the prominent part of the concave steel. The exciter adopts elastic suspension, vertical excitation, excitation rod ends a force sensor. Fig. 4 for the exciter placed in kind.

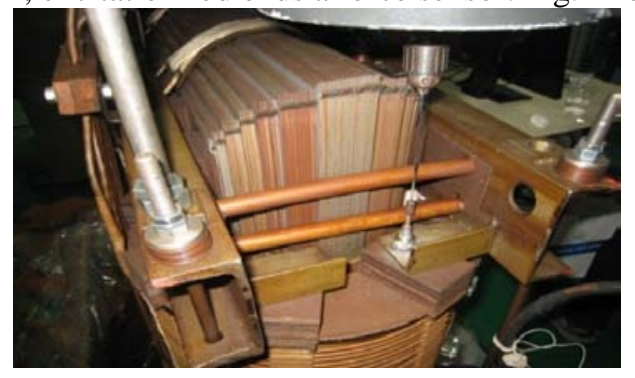

Figure 4. The vibrator is placed in the physical diagram

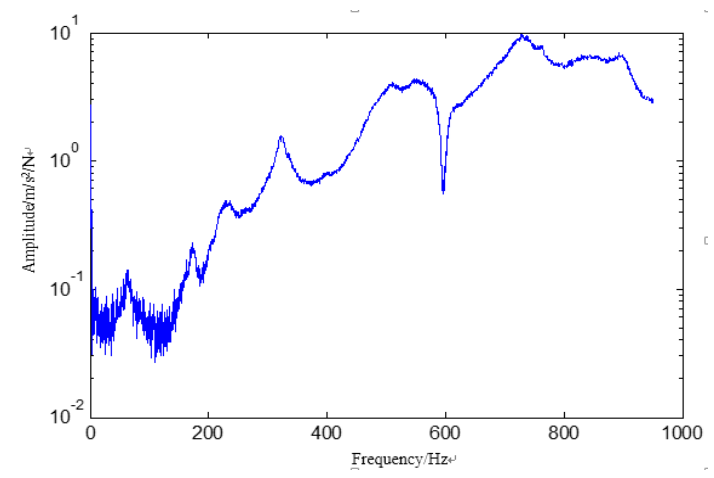

Figure 5. The A phase winding frequency response function 
Fig. 5 is a phase winding axial experimental modal testing the vibration signal frequency response function is the result of the superposition, using a semi logarithmic coordinate representation. You can see from the figure, the frequency response function have obvious peak superposition results in $229 \mathrm{~Hz}$, $322 \mathrm{~Hz}, 501 \mathrm{~Hz}$. Using the PolyMAX method to identify the natural frequency of the A phase winding, the first 4 order natural frequency of the winding is obtained as shown in Table 1. Tab. 2 and Fig. 6 gives the MAC values of the natural frequencies of the different frequencies and the MAC diagrams. By chart shows, the A phase winding under normal operating conditions, the first 4 order of the axial vibration natural frequency difference is larger, and are far away from the excitation frequency of the winding is 2 times that of $100 \mathrm{~Hz}$, the structure is more reasonable. At the same time, the MAC value of the 2 order modes can be considered to be effective in extracting the natural frequency of the 4 order vibration.

Table 1. Four order natural frequency of winding

\begin{tabular}{|c|c|c|c|c|}
\hline Order & 1 & 2 & 3 & 4 \\
\hline Frequency(Hz) & 230 & 322 & 510 & 724 \\
\hline Damping ratio(\%) & 1.71 & 0.62 & 1.70 & 1.54 \\
\hline
\end{tabular}

Table 2. MAC values of the first four order modes of the A phase winding

\begin{tabular}{|c|c|c|c|c|}
\hline Order & 1 & 2 & 3 & 4 \\
\hline 1 & 1 & 0.06 & 0.04 & 0.06 \\
\hline 2 & 0.06 & 1 & 0.02 & 0.02 \\
\hline 3 & 0.04 & 0.02 & 1 & 0.01 \\
\hline 4 & 0.06 & 0.02 & 0.01 & 1 \\
\hline
\end{tabular}

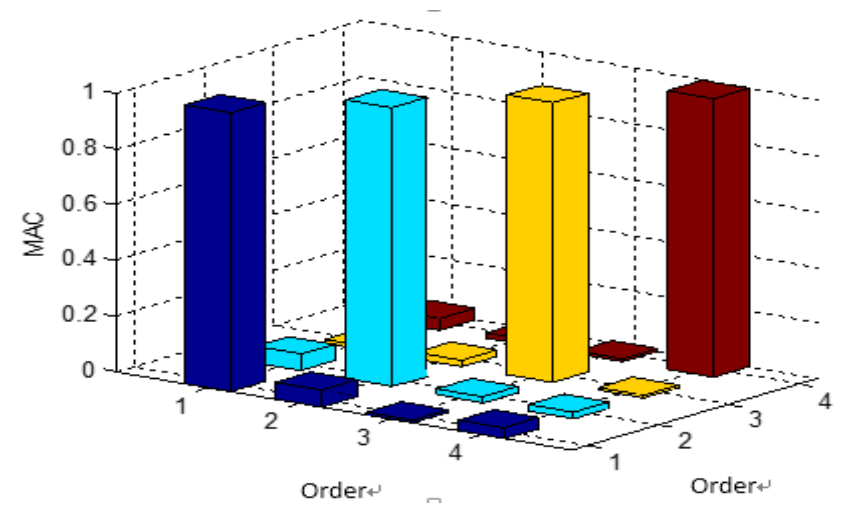

Figure 6. MAC diagram of the first four order modes of A phase winding

\subsubsection{Transformer winding amplitude mode test}

The purpose of the test is to measure the frequency and vibration of the transformer windings and the natural frequency and the corresponding vibration mode of the transformer winding. In the experiment, the sine sweep excitation is used to measure the 20 vibration acceleration sensor. 


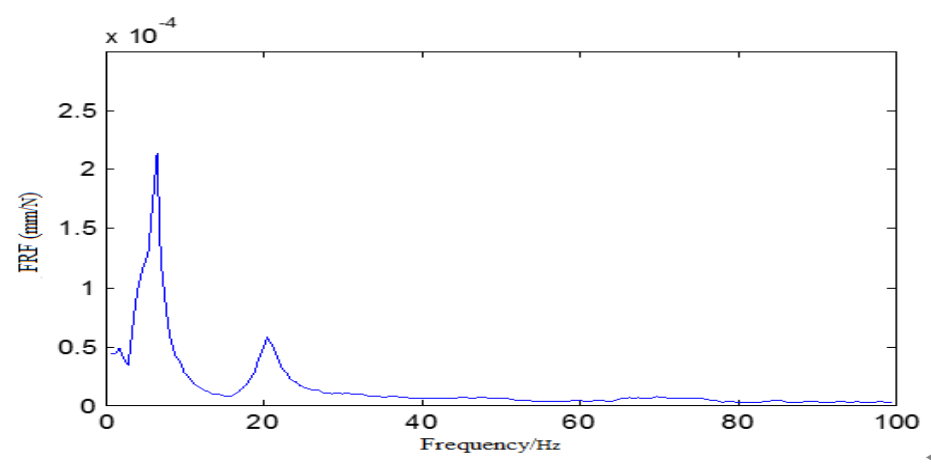

Figure 7. Overall frequency response diagram of winding

The superposition of Fig. 7 for the A phase winding radial modal experiment test the vibration signal obtained by frequency response function. You can see from the figure, the frequency response function have obvious peak superposition results in $6.75 \mathrm{~Hz}$ and $20 \mathrm{~Hz}$. Using the PolyMAX method to identify the natural frequency of the A phase winding, the first 3 order natural frequency of the winding is obtained as shown in Table 3. Table 4 and figure 8 gives the MAC values of the natural frequencies of the different frequencies and the MAC diagrams. By chart shows, A phase winding under normal operating conditions, the first 2 order of the natural frequency of the frequency difference is larger, and are far away from the excitation frequency of the winding is 2 times that of $100 \mathrm{~Hz}$, the structure is more reasonable. At the same time, the MAC value of the 2 order modes can be considered to be effective in extracting the natural frequency of the first 2 order vibration.

Table 3. Two order natural frequency of winding

\begin{tabular}{|c|c|c|}
\hline Order & 1 & 2 \\
\hline Natural frequency(Hz) & 6.75 & 20 \\
\hline Damping ratio(\%) & 5.22 & 4.79 \\
\hline
\end{tabular}

Table 4. MAC values of the two order mode of the winding

\begin{tabular}{|c|c|c|}
\hline Order & 1 & 2 \\
\hline 1 & 1 & 0.32 \\
\hline 2 & 0.32 & 1 \\
\hline
\end{tabular}

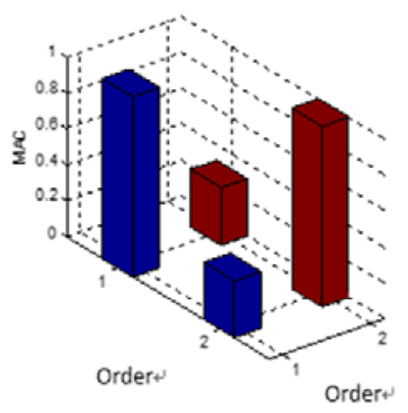

Figure 8. MAC diagram of the first two order mode of winding

\section{Conclusion}

In this paper, the vibration test of the transformer windings is carried out. The PolyMAX method is used to identify the modal parameters of the transformer windings. The 4 order natural frequency and 
damping ratio of the transformer windings are extracted. The MAC value and the MAC chart are introduced. Experimental results show that the modal parameter identification based on PolyMAX method has good noise resistance, and its accuracy is higher than that of the traditional frequency domain identification method. At the same time, because of the experimental transformer winding is a pie coil structure, and large power transformer winding structure is same, so this paper is also used in $220 \mathrm{kV}$ and $110 \mathrm{kV}$ and other high voltage grade power transformer winding modal parameters identification, identification results can provide basis for transformer design, manufacture and vibration based winding state detection.

\section{References}

1. SHAO Yuying, XU jian, RAO Zhushi, et al. Application reseach of detection transformer windings' deformation based on vibration frequency response analysis[J]. Journal of Shang hai Jiaotong University, 44 (9):1223-1228, (2010).

2. GUO jian, LIN Heyun, XU Zihong, et al. Analysis of axial stability of power transformation windings using finite element [J]. High Voltage Engineering, 33 (11):209-212, (2007).

3. LI Yan, ZHOU Wei, JING Yongteng, et al. Axial vibration analysis of transformer active part under short circuits [J]. Advanced technology of Electrical Engineering and Energy, 31(3):49-53, (2012).

4. WANG Hongfang, WANG Naiqing, LI Tongsheng. Axial vibration equivalent one degree analysis of power transformer winding under short circuit [J]. Transformer of China Electrotechnical Society, 15 (5):39-41, (2000).

5. LI Hongkui, LI Yan. Axial vibration modal analysis of transformer windings under different level of precompression[J] Electric Machines And Control, 14(8): 98-101,(2010).

6. Brat Peeters, Heram Van der Auweraer, Patrick Guillaume, et al. The PolyMAX frequency domain method: A new standard for modal parameter estimation [J]. Shock and Vibration, 11:95409, (2004).

7. Carmona R, Hwang W L, Torresani B. Multi-ridge detection and time frequency reconstruction[J]. IEEE Transaction on Signal Processing, 1999(47):480-492.

8. Peeters B, Guillaume P. Automotive and aerospace applications of the LMS PolyMAX modal parameter estimation method[C]//Proceedings of the 22th International Modal Analysis Conference,Dearborn,USA,January,(2004).

9. Guillaume P, Verboven P, Vanlanduit S, et al. A poly-reference implementation of the leastsquares complex frequency domain estimator[C]//Proceedings of the 21th International Modal Analysis Conference. Kissimmee, USA, February, (2003).

10. [10] GENG Chao, WANG Fenghua, HUANG Hua, et al. Transformer Winding Modal Parameter Identification Based on Complex Morlet Wavelet Transform [J]. Journal of Shanghai Jiaotong University, 47 (12):1981-1986, (2013). 